apatinib combined with chemotherapy plus PD-1 antibody drug (2/26), and apatinib combined with PD-1 antibody drug (9/26) and apatinib combined with chemotherapy plus radiotherapy (1/26). The median follow-up time was 5 months. The ORR and DCR were 47.3\%(9/19) and 94.7\%(18/19). The median PFS was not reached.

In addition, 23 pts with ovarian cancer were enrolled in this study. Among them 15 pts were platinum-sensitive, 8 pts were platinum-resistant. The treatment regimens were: apatinib combined with chemotherapy (19/23), apatinib monotherapy (1/23), apatinib combined with chemotherapy drugs plus PD-1 antibody drug (1/23), apatinib combined with PD-1 antibody drug (1/23) and apatinib combined with PARPi (1/23). The median follow-up time was 6 months. The median PFS was 4 months.The ORR and DCR was $14.3 \%(3 / 21)$ and $85.7 \%(18 /$ 21).

In this real-world study, the incidence of adverse reactions of ovarian cancer and cervical cancer was 79.6\%. Grade 3 neutropenia, leukopenia, anemia, proteinuria and thrombocytopenia were observed in 2, 1, 5, 2 and 3 pts.

Conclusion* In this real-world study, apatinib showed a favorable efficacy and safety profile in pts with gynecological cancer. It might lead to better survival benefit in treatment for gynecological cancer pts.

\section{COVID-19 VACCINATION-ASSOCIATED PITFALLS ON TREATMENT RESPONSE EVALUATION WITH CT SCANS OVARIAN CANCER PATIENTS}

G Mancebo Moreno*, A Taus, L Fernandez-Sanahuja, S Espuelas, E Miralpeix, J Castella, B Fabrego, A Salvado, J Sole-Sedeno. Hospital del Mar, Gy, Barcelona, Spain

\subsection{6/ijgc-2021-ESGO.279}

Introduction/Background* Some studies have highlighted the imaging finding of vaccine-associated lymphadenopathies postCovid19 vaccines. That may have a direct impact on the diagnostic accuracy of oncologic patients. Therefore, imaging experts suggest postponing imaging explorations to 6 weeks away from vaccination. Nevertheless to postpone imaging can interfere in assessment of disease extent or clinical response of these patients. We aimed to emphasize the relevance of these findings in ovarian cancer (OC) patients.

Methodology We report three cases of asymptomatic OC patients that presented enlarged lymphadenopathies in CT scans and who have received at least one dose of mRNA Covid-19 vaccine at 6,8 and 28 days prior to the CT examination, respectively.

Result(s)* Two of them were considered as having benign vaccine-associated lymphadenopathies as they presented right supraclavicular and intrapulmonary lymphadenopathies with no other clinical findings, nevertheless a new CT scan was performed 3 months later to reassure diagnostic. The third patient, one year after being diagnosed by EOC, was diagnosed by Lymphoma. After finishing treatment, a PET/CT SCAN to response evaluation was performed, 6 days after of COVID-19 vaccination. All tumoral nodes except one hypermetabolic lymph located at the lateral side of left Iliac Artery disappeared. This lymph node was considered as either no responsive or relapsed from OC, and less likely vaccine-associated. After being resected a high grade carcinoma was found.
Conclusion* The incidence of lymphadenopathies in vaccinated patients raises a challenge in the interpretation of imaging of OC patients. Present cases emphasize the need for an accurate clinical evaluation encompassing the type and timing of Covid-19 vaccination. This would also allow avoiding imaging misinterpretations and unnecessary further assessment. Evidence-based guidelines are needed as postponing imaging controls to 6 weeks away from vaccination may be problematic in assessment of clinical response in treated OC patients or newly diagnosed patients.

The authors declare no conflicts of interest. None received grant support for this study neither for related clinical or research activity

\section{VESSEL INJURY AND REPAIR}

M Zikan*, O Dubova, V Student, P Koliba, T Brtnicky. Charles University - First Faculty of Medicine and Bulovka University Hospital, Dept. of Gynecology and Obstetrics, Praha 8, Czech Republic

\subsection{6/ijgc-2021-ESG0.280}

Introduction/Background* Vessel injury is a common event in retroperitoneal procedures and technique of vessel injury repair should be a part of gynecological oncology education.

Methodology Video presentation of a) a point injury to external iliac vein and technique of suture as an example of common surgical complication and way of solution b) large damage to vena cave and repair with vascular prosthesis as an example of rare and potentially catastropic complication

Result(s)* Two surgical complication - vessel injuries - were managed step-by-step with intention to reduce the blood loss and repair vessel to prevent long-term complications as a vein thrombosis.

Conclusion* Knowledge of appropriate surgical vessel repair techniques protects against long-term postoperative complications. Even dramatic vessel injury can be repaired with a sequence of steps preventing massive hemorhage and using vesseel prosthesis.

\section{OCCULT CARCINOMA IN BREAST RISK REDUCTION SURGERY IN BRCA MUTATION CARRIERS}

${ }^{1} \mathrm{~J}$ Montero Olmeda, ${ }^{2} \mathrm{MDLR}$ Oliver ${ }^{*},{ }^{2} \mathrm{~A}$ Laura, ${ }^{3} \mathrm{E}$ Felipe Pardo, ${ }^{3} \mathrm{M}$ De Miguel Reyes, ${ }^{1} \mathrm{G}$ Marta, ${ }^{3} \mathrm{~S}$ Aragon. 'Hospital 12 de Octubre, Ginecología y Obstetricia, Madrid, Spain;

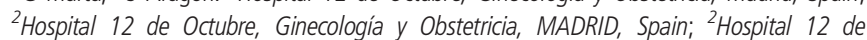
Octubre, Ginecología y Obstetricia, MADRID, Spain

\subsection{6/ijgc-2021-ESG0.281}

Introduction/Background* Breast cancer is one of the most frequent tumors in the female population and can be associated with the detection of pathogenic germ-line BRCA1/2 mutation, which leads to close monitoring of patients and the consideration of prophylactic surgery. Occult synchronous breast cancer occurs in approximately $1-3 \%$ of patients and is detected incidentally.

The aim of our study is to determine the incidence of occult breast carcinoma in patients with BRCA $1 / 2$ carriers who accept prophylactic mastectomy as risk reduction surgery. Methodology Retrospective analysis of all patients assessed in the Breast Pathology Unit of the 12 de Octubre Hospital 